Cahiers de recherches médiévales

Journal of medieval studies

$4 \mid 1997$

Être père à la fin du Moyen Âge

\title{
La paternité à l'aube de l'an 2000
}

\section{Geneviève Delaisi de Parseval}

\section{OpenEdition}

Journals

Édition électronique

URL : https://journals.openedition.org/crm/975

DOI : $10.4000 / \mathrm{crm} .975$

ISSN : 1955-2424

Éditeur

Honoré Champion

Édition imprimée

Date de publication : 15 décembre 1997

ISSN : 1272-9752

Référence électronique

Geneviève Delaisi de Parseval, «La paternité à l'aube de l'an 2000 », Cahiers de recherches médiévales [En ligne], 4 | 1997, mis en ligne le 15 janvier 2007, consulté le 15 décembre 2022. URL : http:// journals.openedition.org/crm/975; DOl : https://doi.org/10.4000/crm.975

Ce document a été généré automatiquement le 15 décembre 2022.

Tous droits réservés 


\title{
La paternité à l'aube de l'an 2000
}

\author{
Geneviève Delaisi de Parseval
}

1 Après lecture attentive des articles de ce numéro consacré à la paternité au Moyen-Age, plusieurs réflexions viennent à l'esprit d'une psychanalyste qui réfléchit depuis une vingtaine d'années aux nouvelles définitions et déclinaisons de la paternité.

2 En premier lieu, l'article de Danièle Alexandre-Bidon montre que, contrairement au cliché largement répandu selon lequel les pères ne s'intéresseraient pas à leurs enfants avant l'âge de la marche ou avant celui de l'entrée à l'école, les pères médiévaux «maternaient», s'occupaient des nourissons, donnaient le biberon; bref s'avéraient experts en puériculture...; jusqu'à, dans un cas sans doute significatif, se faire représenter au lit, "en couvade», le nouveau-né dans les bras, s'apprêtant à consommer le bouillon de poule de l'accouchée! On n'est pas très loin de l'image du «nouveau père » largement répandue dans les années quatre-vingt, père qui changeait son bébé, faisait les courses avec lui, ce dernier sanglé contre son ventre dans un sac kangourou. Image qui a fait long feu à la fin des années quatre vingt-dix pour être remplacée par des clichés plus «intersexués » où les représentations de pères et de mères semblent devenus interchangeables, mais aussi sans définition spécifique, sans repères pourrait-on dire! Retournements dans les représentations du père dans leur façon de s'accommoder les bébés, virages à $180^{\circ}$ de la puériculture, qui, on le voit, ne datent pas d'aujourd'hui...

3 Si dans la société du début du $\mathrm{XV}^{\mathrm{e}}$ siècle se développe la recherche d'une nouvelle conception -ou d'une redéfinition- de la paternité autour de questions qui mettent en cause les fondements même de la société médiévale (article de Paul Payan), on constate que le questionnement de la fin du millénaire suivant -le nôtre- en renvoie un écho étrangement ressemblant. Symptomatique de cet écho est la comparaison souvent faite entre l'image de Joseph à l'époque médiévale et celle, contemporaine, de l'homme qui devient père au cours de procréations artificielles avec dons de gamètes masculins (IAD ou FIV-D ${ }^{1}$ ). Comme Joseph en effet, le «père-IAD » est, on le verra plus loin, père légal, père nourricier et protecteur, mais sans lien naturel avec son enfant -puisqu'il y a participation d'un donneur de sperme-; en situation d'infériorité par conséquent, à l'instar de Joseph, tant vis à vis de son enfant que de sa compagne. Comme pour Joseph, 
le statut du père avec dons de gamètes, tel qu'il est organisé par les lois bioéthiques de juillet 1994, se situe au croisement de deux traditions : l'une, fondée sur le droit romain, permet l'adoption (adoption de l'enfant du conjoint, mais adoption également par le père social des gènes donnés par le père biologique); l'autre, plus ancienne (tradition juive? ou remontant à Origène?), se fonde sur les liens du sang : ceux-ci apparaissent tellement important de nos jours, tellement "fondateur de filiation", que la société cherche à tout prix à gommer la participation d'un géniteur autre que le père social ; les lois bioéthiques rattachent ainsi, au plan juridique, la filiation artificielle à la filiation naturelle, faisant, de ce fait, définitivement disparaître le donneur de gamètes.

Dans la préface de ce numéro, Didier Lett donne la définition du père au Moyen-Age : «Être père, c'est se référer à des modèles, avoir un certain pouvoir, aimer et éduquer un enfant, donner son nom, infuser sa culture, produire des ressemblances ». On verra dans les pages qui suivent que les changements contemporains continuent de se décliner sur le même mode, et à partir de questionnements voisins. Le thème des marqueurs de ressemblance en particulier donne à voir, comme au microsocope, deux théories opposées sur le rôle du père et de la mère dans la «fabrication» d'un enfant. On sait que l'une des deux «théories», dans la tradition médicale de l'aristotélicisme, confère au sperme masculin le pouvoir de former et de modeler l'enfant, la mère ayant essentiellement un rôle de "portage », d'« hôtel-restaurant » si l'on veut bien accepter ce barbarisme.. ${ }^{2}$. Le rôle de "restaurant» étant «tenu» par le sperme du père transformé par la mère en nourriture pour le foetus pendant la grossesse d'une part, par le lait maternel, vecteur lui aussi de ressemblance, de l'autre. Quant à la seconde théorie, plus habituelle dans l'imaginaire moderne, elle accorde à la mère un rôle, «naturellement » plus important que celui du père dans la fabrication de l'être humain. Ces deux théories, dit Didier Lett, coexistent au Moyen-Age, sources d'oscillations entre deux critères de filiation. Or cette problématique de la désignation : "qui est le père?", "qui est la mère?» est au cœur des débats actuels qui se sont noués autour des transformations contemporaines de la parentalité et la famille. Et ceci tant à propos de l'augmentation des divorces et de l'apparition des familles dites «recomposées", qu'autour du phénomène médico-social des procréations médicalement assistées que nous analyserons, lui, en détail. Le critère d'une paternité fondée sur la volonté, dans le droit fil du droit romain, revient en force en effet dans la désignation des parents qui deviennent parents grâce à des dons de gamètes ou d'embryons. Quant au critère fondé sur les liens du sang, sur «le biologique » comme on dit maintenant, il a aussi une part très importante à l'heure actuelle, ne serait-ce qu'à travers la preuve juridique de la paternité, à partir du sang du père précisément!

5 Ces quelques commentaires éveilleront, nous l'espérons, un écho à la lecture des pages qui suivent qui tentent d'analyser les représentations de la paternité dans cette fin de siècle.

Qu'on nous permette, avant d'en arriver à la paternité contemporaine, quelques considérations rapides sur un épisode de l'histoire de la paternité «à la française » qui intéresse évidemment beaucoup les psychanalystes. Les Français peuvent en effet se targuer d'être un des rares peuples de l'Histoire à avoir pratiqué, à la Révolution, ce qu'on peut appeler un "parricide monarchique ", véritable parricide à l'échelle sociale. Il s'est agi là de la première grande rupture dans l'affaiblissement progressif du pouvoir du père en France depuis le pater familias du droit romain. C'est dans ce cadre que 
l'exécution de Louis XVI en août 1793 a pris une forte portée symbolique. Balzac a justement écrit : «En coupant la tête de Louis XVI, la République a coupé la tête à tous les pères de famille». (Lettre 12, Mémoire de deux jeunes mariés). Cet acte, symbolique et réel à la fois, fût, on le sait, suivi d'un droit révolutionnaire, qui, en l'espace de trois ans, ôta tout pouvoir aux pères. Quelques années plus tard, rien ne subsistait de la patria potestas de l'Ancien régime; le siècle des Lumières avait sonné le glas de la puissance paternelle. Au cours du $19^{\circ}$ siècle, la toute-puissance du père sera peu à peu peu émiettée ; le siècle s'achèvera sur une crise générale de l'identité masculine dont la paternité n'est d'ailleurs qu'un des aspects. ${ }^{3}$

7 Cette perte progressive de puissance, en tant que phénomène social, a produit un ensemble d'effets idéologiques encore actuels concernant les pères. La principale caractéristique est une accusation généralisée de faiblesse et d'impuissance portée à leur encontre: il seraient absents, défaillants, classiquement stigmatisés comme «carents $»^{4}$. Ces notions conjointes de perte de puissance et de carence ont engendré les questions contemporaines sur la définition du père : un père qui n'est ni "puissant ", ni "chef de famille" peut-il être père quand même ? Ou, de manière symétrique et inverse : «Un père qui «paterne » (qui change le bébé, qui le berce, fait les courses avec lui) sur le modèle du rôle maternel, peut-il être un vrai père ? Un père qui reste à la maison tandis que sa compagne travaille à l'extérieur est-il un "père suffisamment bon", comme aurait pu le dire le pédiatre anglais Winnicott? Ces questions, légèrement caricaturales, sont néanmoins symptomatiques d'une angoisse devant un important changement dans les mentalités. Elles ont alimenté en France, depuis les années 80 , un véritable débat de société autour de la question des «nouveaux pères ». Selon Daniel Roche, nous sommes entrés dans la seconde révolution des pères. ${ }^{5}$

De nouvelles formes de paternité, liées aux différents cas de figure de l'assistance médicale à la procréation ${ }^{6}$, sont apparues au cours des deux dernières décennies. Ces techniques ont eu un rôle important dans les nouvelles représentations de la parentalité, dans la mesure où elles ont favorisé une distribution radicalement nouvelle des rôles parentaux face à la procréation.

9 C'est donc ce microscope que nous allons utiliser pour faire un "point épistémologique » sur la part contemporaine du père en cette fin de siècle.

Plusieurs caractéristiques se dégagent de la paternité en AMP :

11 1 - la paternité, désormais médicalisée et médiatisée, est devenue additionnelle, »éclatée » entre plusieurs individus (hommes ou institutions). On assiste en effet en AMP à une disjonction entre plusieurs hommes des fonctions du père : clivage entre géniteur et père essentiellement; mais on compte aussi, dans les autres figures paternelles, le médecin, la «banque du sperme », également le juge ou le notaire (qui recueillent les consentements des participants dans les cas où il $\mathrm{y}$ a intervention d'un autre homme, le donneur). Dans le cas de l'insémination artificielle par donneur IAD-, ou en fécondation in vitro avec donneur de sperme -FIV-D-, un conjoint infertile peut, en AMP, devenir père d'un enfant, tandis que le géniteur anonyme de cet enfant (appellé, par convention, "donneur) ", fécondera, lui, par la double médiatisation de son sperme mis en paillettes et congelé, et de l'intervention d'un médecin, l'épouse ou la compagne du mari infertile, père potentiel. Il s'agit, en somme, d'un cumul de paternités, avec apparition d'une paternité «additionnelle» dans laquelle plusieurs « co-géniteurs »-ou « co-pères »-participent à la conception d'un enfant. 
122 - La paternité est devenue intentionnelle et adoptive, mais dans un sens particulier : il s'agit d'une adoption pré-conceptionnelle. Il s'agit soit d'une adoption par le père social des gènes qui ont été donnés par un autre homme à son futur enfant, soit de l'adoption d'un embryon donné par un autre couple, soit enfin de l'adoption pré-conceptionnelle de l'enfant du conjoint ${ }^{7}$.

133 - La paternité est devenue différée ou différable : ce n'est plus, dans de nombreux cas d'AMP, le moment de la fécondation qui détermine l'âge de la conception, mais, désormais, celui de la décongélation des gamètes. C'est ce à quoi on assiste par exemple dans les cas suivants :

14 - dans les cas d'hommes vasectomisés qui recourent ultérieurement à une IAC (insémination avec le sperme du conjoint, dite insémination homologue) avec des paillettes de leur propre sperme qui avait été préalablement congelé,

15 - dans les cas d'hommes qui ont subi un traitement stérilisant (en général en raison du traitement d'un cancer) et recourent ultérieurement, quand ils sont guéris, à une IAC.

16 - dans les cas de paternité où à la suite d'une FIV, le couple a des embryons congelés, dits «surnuméraires ", qui seront éventuellement réimplantés des années plus tard.

174 - La paternité est devenue certaine: on a, peu à peu, assisté à une sorte de renversement de l'adage latin mater semper certa est, sed pater semper incertus. La paternité est désormais affaire de preuve, comme l'a montré un jugement célèbre (1976) de la Cour de Paris. Comme le montre aussi l'exemple de l'ICSI ${ }^{8}$, technique récente d'AMP, qui consiste à injecter, au cours d'une FIV, dans l'ovocyte, un spermatozoïode du compagnon ou mari. Preuve imparable d'une paternité biologique...

18 Mais cette certitude de la paternité par AMP s'accompagne, paradoxalement, d'une grande fragilité, le père s'étant, pour ainsi dire, auto-proclamé. La filiation en AMP repose exclusivement en effet sur la volonté : il n'y a pas de jugement, à la différence de l'adoption, et le mariage n'est pas une condition nécessaire à l'acceptation de la demande d'AMP (plus de pater is est par conséquent). Mais cette facilité a un revers de médaille. La loi actuelle rend en effet excessivement fragile la paternité du père en IAD : la filiation avec participation d'un tiers donneur est en effet contestable : "s'il est soutenu que l'enfant n'est pas issu de la procréation médicalement assistée, ou si le consentement a été privé d'effet $»^{9}$. On peut déjà anticiper les contentieux qui s'ouvriront... La seule paternité irréfutable serait celle que conférerait au père stérile l'adoption légale de l'enfant de sa compagne, au terme d'un jugement. En outre, depuis le vote des lois sur l'AMP, l'existence même de la filiation avec participation de donneurs fait l'objet d'un déni social et juridique, le législateur français ayant choisi de rattacher la filiation « artificielle » à la filiation légitime ou naturelle. Au lieu d'opter, ce qui aurait été un autre choix possible, pour un rattachement de la filiation par AMP à la filiation volontaire, telle celle de la filiation adoptive. Solution pourtant plus cohérente aux yeux de nombreux juristes ${ }^{10}$, et sans doute plus respectueuse pour les différents protagonistes. Ce choix du législateur indique en tous cas clairement un refus social d'admettre une quelconque dissociation ou cumul de la fonction paternelle. Il signe une volonté de substitution d'une filiation à une autre (on dissout l'une pour y substituer une autre); on détruit les traces de l'homme qui a donné ses gamètes, en faisant croire à une engendrement qui n'a pas eu lieu. ${ }^{11}$ Le tout ayant lieu sous la houlette du juge ou du notaire, en secret (comme à la confession?). 
19 Et pourtant... Il est intéressant de rappeler que l'adoption n'a été conçue comme remplacement de la filiation d'origine que depuis 1939. Avant cette date, la seconde filiation s'additionnait à la première. On sait aussi que que l'adoption simple, largement sous utilisée de nos jours, procède par additions de filiations. De manière significative, la clinique de l'IAD montre d'ailleurs que, plus encore que le questionnement sur l'identité du donneur de sperme stricto sensu ${ }^{12}$, c'est davantage la stérilité du père, le deuil non fait par le père -ou par le couple- de sa fertilité qui obére la dynamique de ces familles (parents et enfants). Et, en l'espèce, le travail de deuil de la fertilité du père et du couple n'est pas facilité, loin de là, par la possiblité du déni sur le fait même d'avoir recouru à un don de gamètes. Rêvons un peu: essayons, en ayant une pensée pour le destin du malheureux Oedipe, de nous placer du côté de l'enfant. Pourquoi, au fond, l'enfant né par IAD n'aurait-t-il pas le droit de connaître la particularité de son histoire (tout à fait digne au demeurant) : savoir que son père, celui qui l'élève et dont il porte le nom, son père affectif et effectif, n'est pas son géniteur? Savoir qu'il existe un autre homme, un «oncle d'Amérique " pourrait-on dire, personnage additif, "héros positif seulement ", qui a participé à sa venue au monde ? La particularité du montage de l'IAD, fabriqué, inventé de toutes pièces par les institutions médicales et juridiques de cette fin de siècle, ne devrait-il pas au moins, en toute équité, accorder aux enfants ainsi conçus une filiation noble, filiation qui serait fondée sur le cumul des paternités et non sur la substitution de l'une à l'autre? Comme c'est le cas de la filiation adoptive. Outre l'interêt bien compris de l'enfant, la prise en compte d'une pluripaternité respecterait à la fois la paternité du père affectif et social (en lui facilitant précisément le travail de deuil de sa fertilité), mais ausi le psychisme de l'homme qui donne son sperme ${ }^{13}$, de cet homme, de cet autre père, qui devrait être considéré autrement que sur le mode d'un étalon donnant sa semence... Les donneurs de sperme, pères de famille en général, ont, eux aussi, une histoire...

Il y aurait beaucoup à dire, au plan psychanalytique, sur la paternité en AMP. Insistons seulement ici sur un point assez méconnu. De quoi s'agit-il, en réalité, dans ce "passage du témoin » d'un homme à un autre, dans cette dynamique paternelle qui se joue entre deux hommes, via une femme? En IAD ou FIV-D, il y a en effet, on le voit, don de sperme de la part d'un homme (le "donneur d'hérédité », selon l'heureuse expression du doyen Carbonnier) à un autre homme (le père social); ceci par le truchement du corps d'une femme, la future mère ${ }^{14}$. Il y a là, à notre sens, une donne possiblement incestueuse qui pourrait évoquer un «inceste du deuxième type $»^{15}$, inceste entre deux femmes et/ou entre deux hommes ${ }^{16} \mathrm{La}$ définition de l'inceste du deuxième type est la suivante: c'est un inceste entre individus de même sexe qui ne sont pas homosexuels mais partagent le même partenaire sexuel. Selon l'analyse qu'en fait Françoise Héritier, cette rencontre transgresse l'impossibilité de faire se rencontrer des substances considérées comme de nature identique; l'interdit du même sur le même par conséquent. Cette hypothèse est corroborée tant par l'analyse de textes littéraires que par celle de la clinique psychanalytique: le partage d'une même femme par deux hommes réalise, dans le fantasme, un rapport homosexuel (entre le mari et son rival...) et, ce, sous le déguisement d'un rapport hétérosexuel. Ce qui n'est pas sans évoquer le cas de l'AMP (au sens des relations fantasmatiques entre le donneur de sperme et le père). Remarquons tout de même, et afin de ne pas nous faire accuser de diaboliser l'AMP, que ce fanstasme incesteux du «deuxième type » est, dans l'IAD, relativement bien tenu à distance, canalisé, endigué, du fait qu'il n'y pas de relations sexuelles 
vécues directement entre la mère et le donneur de sperme. Il y a seulement -mais ce n'est ni neutre ni anodin- rencontre de substances dans le même "lieu»: le vagin de son épouse dans lequel le sperme du mari «rencontre » le sperme du donneur.

Fermons ici la parenthèse de la paternité en AMP et venons-en à une approche psychodynamique du devenir père: hommes et femmes sont-ils, au fond, si différents face à cette étape essentielle de la vie, qu'est le «devenir-parent »? De notre point de vue, l'expérience de la paternité peut, en bonne logique métapsychologique -et pour paradoxal que puisse sembler ce propos-, se comprendre comme une série d'étapes psychiques analogues à celles de la maternité, bien qu'elles soient à l'évidence inscrites différemment dans le corps du père et dans celui de la mère. Mais notre expérience clinique d'écoute des conjoints ou de femmes enceintes suivis pendant la grossesse de leurs compagnons nous a convaincue qu'il existait, chez les pères ou futurs père, une «transparence psychique» analogue à celle qu'on a classiquement remarquée chez la mère pendant la période péri-natale. Comme une sorte de contagion de l'état affectif de la mère et du père, au bébé. Transparence psychique qui facilite la communication avec des niveaux archaïques du psychisme et peut conduire à des régressions ainsi qu'à des retours à des points de fixation du développement libidinal. Rappellons ici la variété des symptômes de couvade qui, loin d'être une coutume propre aux sociétés traditionnelles ou exotiques, traduit chez nous comme ailleurs, les vicissitudes du devenir-père. On connait également les «folies paternelles", décompensations psychotiques chez certains hommes qui se produisent à l'occasion d'une paternité. Il existe enfin des symptômes typiques de l'accès à la paternité que certains cliniciens américains ont appelé les «trois $\mathrm{F}$ »: fear, flight, fight (angoisses, fuites, comportements violents ou agressifs). Sans compter un certain nombre d'acting out dans le domaine de l'activité sexuelle. Ces troubles psychologiques ou psychosomatiques ne surviennent évidemment pas par hasard dans l'histoire des sujets qui les vivent: les pères qui en sont atteints sont peut-être psychiquement plus fragiles et mal préparés à aborder le processus du devenir-père. Ceux, notamment, que nous avons appelés "primipères" (par analogie avec les primipares) ${ }^{17}$ vivent une paternité dont l'approche mobilise une fantasmatique, défensive le plus souvent, qui tourne autour d'un certain nombre de pôles conflictuels : conflits vis-à-vis de la future mère et du bébé bien sûr, mais aussi vis-à-vis de la fratrie du père, enfin et surtout peut-être vis-à-vis des parents (de son propre père, en particulier). Cette psychodynamique est, au demeurant, bien connue dans la clinique de l'infertilité masculine et de ses réparations -celle de l'IAD en particulier- qui renvoie à une problématique complexe de l'homme stérile vis-à-vis de son propre père, en même temps qu'elle l'enferme dans un secret, en général électif, vis-à-vis de lui.

La façon d'envisager la paternité telle qu'elle est présentée ici insiste, on le voit, sur une dynamique, un fonctionnement psychique parallèle, à bien des égards, entre l'homme et la femme au regard de l'accès au devenir-parent. Un bref rappel s'impose sur un aspect des travaux de la psychanalyste américaine Thèrèse Benedek -moins connu que ceux qui concernent la maternalité- qui éclaire la compréhension de la part psychique chez le père dans l'accès au devenir-parent ${ }^{18}$. Benedek a montré qu'au regard de la procréation l'homme et la femme ont un fonctionnement psychique équivalent. La «crise» de la paternité (au double sens de crise -clastique- et d'intégration des pulsions) fait régresser l'homme à une phase antérieure de sa maturation libidinale. De plus, favorisé culturellement par la vie en couple, ce mouvement s'intègre aux 
mouvements régressifs d'identification de l'homme à sa compagne pendant la grossesse de cette dernière. Partageant fantasmes et projections de la future mère, l'homme en puissance de paternité revit ses identifications respectives avec ses propres père et mère pendant son développement. Enfin, les premières relations père/bébé réactivent l'amour maternel du père qui éprouve à cette occasion une nouvelle gratification libidinale (il est un «bon père/ bonne mère »).

Cette vision métapsychologique de la paternité a, entre autres interêts, celui de faire "décoller» la question du père du sempiternel amalgame entre père et fonction paternelle, entre père et rôle paternel. La théorie lacanienne $\mathrm{du}$ "nom-du-père » $\mathrm{a}$ en effet quelque peu servi d'écran face à la question du père réel. Et il y a, à notre sens, un peu de trop de symbolique et pas assez de chair dans cette approche. ${ }^{19}$

Ce n'est en effet pas du père symbolique -cher aux lacaniens- dont il est question ici, mais du conjoint ou compagnon de la future mère; du père réel, charnel, aux origines, lui aussi, du futur sujet humain. Nous nous référons à une clinique que nous connaissons bien, celle de la paternité des conjoints (maris ou compagnons, engendreurs ou non) de la future mère ; à de vraies histoires de pères qui mettent un enfant au monde et se reconnaissent comme pères; à la clinique des pères de la triade père/mère/bébé, de ces pères -peut-être "nouveaux", à moins qu'il ne soient nouvellement reconnus?-qui, ont auprès des nourissons et des bébés, un rôle extremement précoce. ${ }^{20} \mathrm{À} \mathrm{ces} \mathrm{pères} \mathrm{capables} \mathrm{de} \mathrm{contenir} \mathrm{des} \mathrm{angoisses} \mathrm{de} \mathrm{la} \mathrm{mère} \mathrm{;} \mathrm{à}$ ces pères qui sont devenus pères en fonction du regard de leur propres pères sur eux ; bref à ces pères qui vivent une paternité pas si différente (aussi paradoxal que puisse sembler cette assertion) de la maternité. Dans cette optique métapsychologique, la paternité se comprend non seulement à partir du bébé qu'à ou a engendré le père, non seulement à partir de la relation du père avec la mère de ce bébé, mais aussi à partir de ce qu'enfant il a vécu avec son père et sa mère, sa fratrie. Comme pour la maternité, rien n'est automatique dans la paternité: le fait d'attendre un enfant, d'être un expectant father comme disent les anglo-saxons, crée une potentialité de paternité qui pourra ou non s'actualiser. Mais le fait qu'il existe une réalité du versant psychique de la paternité ne la renvoie pas pour autant, comme cela a été le cas pendant des siècles..., dans le camp -dans le champ- du symbolique, du légal ou du sociologique!

Il existe actuellement, on l'a vu, un flou, une oscillation, dans les repères et dans les représentations de la paternité. Nous sommes de nouveau peut-être dans une zône frontière, époque où l'on passe, presque à sa guise, tantôt du côté de l'ordre biologique, tantôt du coté de l'ordre juridique, tantôt du côté du «psy». Le mystère du devenirpère n'est sans doute pas près d'être totalement éclairci. Mais il n'est pas négligeable que la société soit en passe d'admettre que "ça " $^{21}$ s'inscrit, que «ça» se passe dans le psychisme, pour le père autant que pour la mère. Comme c'était le cas à la fin du Moyen Âge, le monde de la fin du vingtième siècle semble parti, lui aussi, à la recherche de ses pères... 


\section{NOTES}

1. IAD : insémination artificielle avec sperme de donneur. FIV-D : fécondation in vitro avec sperme de donneur. Cf infra p. 135

2. Nous avons tenté d'élaborer ces «théories » dans le chapitre «Les vecteurs de la parentalité ", La part du père, Seuil, 1981.

3. Cf. la conclusion de D. Roche dans J. Delumeau et D. Roche (dir.), Histoire des pères et de la paternité, Paris, Larousse, 1990, pp. 433-445.

4. Cf. G. Delaisi de Parseval et F.Hurstel, «La paternité à la française », Les Temps

Modernes, ${ }^{\circ} 481$, sept. 1986, pp. 51-93.

5. Conclusion de Histoire des pères..., op cit.

6. Désormais désignées par le sigle AMP, depuis les lois dites «bioéthiques» votées le 29 juillet 1994.

7. Dans le cas d'adoption par un mari stérile de l'enfant de sa compagne conçu avec le sperme d'un donneur. cf infra.

8. Intra cytoplasmic spermatozoïd injection.

9. Art. 311-20 de la loi du 29 juillet 1994 sur l'AMP.

10. Par exemple le Pr. Claire Neirinck «Le droit de la filiation et la procréation médicalement assistée ", Les Petites Affiches, ${ }^{\circ}$ 149, 1994, pp. 54-62.

11. Ce qui rappelle étrangement l'histoire d'Oedipe...

12. Et donc sur la filiation biologique.

13. Soldat inconnu de la «légion donneurs »...

14. Dans le cas d'un don d'ovocytes, l'enfant est, à l'inverse, conçu par deux femmes ( grâce à un don d'une femme à une autre), via un homme, le futur père.

15. Selon l'expression de F. Héritier, Les deux sceurs et leur mère, Paris, Ed. O. Jacob, 1994, p. 149 et ss.

16. Analysé par G. Devereux, «Considérations ethnopsychanalytiques sur la notion de parenté » (1956), Ethnopsychanalyse complémentariste ${ }_{2}$ Paris, Flammarion, 1972,

pp. 169-200.

17. La part du père, Seuil, 1981, pp. 117-83.

18. Th. Benedek, «Parenthood as a developmental phase », Journal of American Psychoanal. Ass., n 7, 1959, pp. 389-417.

19. Voir sur ce point l'analyse que donne Gilbert Diatkine, «Mon nom est personne : petite histoire de la forclusion du Nom-du-père », Revue française de psychanalyse ${ }_{2} 1997$, $n^{\circ} 2$.

20. Voir sur ce point les travaux de R. Debray et, notamment, «L'objet en personne : la réalité paternelle ", Revue française de psychanalyse, $1997, \mathrm{n}^{\circ} 2$, pp. 425-34.

21. Le «ça " groddeckien, à la manière dont les couples disent : "ça a marché ", à propos d'une conception recherchée. 


\section{AUTEUR}

\section{GENEVIÈVE DELAISI DE PARSEVAL}

psychanalyste, 118 rue de Vaugirard, Paris $6^{\circ}$. 